\title{
EL ENLACE QUÍMICO: UNA CONCEPTUALIZACIÓN POCO COMPRENDIDA
}

\author{
Riboldi, Liliana ${ }^{1}$; Pliego, Óscar ${ }^{1}$ y Odetti, Héctor ${ }^{2}$ \\ ${ }^{1}$ Universidad Nacional de Rosario \\ ${ }^{2}$ Departamento de Química Inorgánica. Facultad de Bioquímica y Ciencias Biológicas \\ UNL. Paraje «El Pozo». CC 242. Santa Fe (3000) Argentina \\ hodetti @ fbcb.unl.edu.ar
}

\begin{abstract}
Resumen. Este estudio describe las ideas sobre los enlaces químicos de un grupo de estudiantes que iban a comenzar la universidad y las de otro de estudiantes universitarios. Los resultados muestran distintas ideas con respecto a: procesos donde átomos iguales o distintos se agrupan, la posibilidad de que una unión química sea un proceso espontáneo, la estructura de sustancias unidas por enlace iónico o covalente y la causa de un enlace iónico. Resalta también el pobre grado de coherencia de las concepciones manifestada cuando los estudiantes usan una idea en un contexto pero no en otro similar. La investigación encuentra que las concepciones de los alumnos universitarios y preuniversitarios son las mismas en la mayoría de los casos.
\end{abstract}

Palabras clave. Enlaces químicos, uniones químicas, concepciones alternativas, ideas previas, modificación de ideas previas.

Summary. This study describes the ideas about chemical bond of a group of students about to enter university studies and another already at university. The results show different ideas with respect to processes where similar or different atoms group, the possibility of a chemical bond being a spontaneous process, the structure of substances bonding by ionic or covalent bond, and the cause of ionic bond. The study also highlights the poor coherency shown when students use an idea in a certain context but not in another similar to the first. The research finds that the conceptions of university and pre-university students coincide in most cases.

Keywords. Chemical bond, alternative conceptions, preconceptions, modifications of preconceptions.

\section{INTRODUCCIÓN}

El aprendizaje requiere, de acuerdo con el modelo constructivista, la existencia de actividad autoconciente por parte del sujeto como condición para su evolución intelectual, dado que quien aprende interpreta la realidad proyectando sobre ella los significados que va construyendo. La adquisición de conceptos, entonces, no sería otra cosa que un proceso creativo basado en la reestructuración de las teorías de las que dichos conceptos forman parte (Pozo, 1989).

Para este modelo tienen gran relevancia los conceptos y la estructura cognitiva de quien aprende, pues el aprendizaje sólo es significativo cuando puede relacionarse, de modo no arbitrario y substancial, con lo que el alumno ya sabe (Ausubel, Novak y Hanesian, 1978).

La hipótesis de que aprender los contenidos escolares presupone atribuir un sentido y la construcción de sus significados, y que tal reto es enfrentado, en relación genética, sobre la base de los significados que se han construido previamente (Pozo, 1987), resalta la importancia que los conceptos cotidianos de nuestros alumnos revisten en el diseño de cualquier estrategia de enseñanza. Los conocimientos previos no sólo permiten contactar inicialmente los nuevos contenidos, sino que, además, son los fundamentos de la construcción de nuevos significados.

Tales razonamientos han motivado la realización de numerosas investigaciones sobre las concepciones alternativas de los alumnos en los más diversos dominios pero el tema del enlace químico ha sido abordado en pocas investigaciones.

Peterson y Treagust (1989) analizan en estudiantes de 16-17 años conocimientos sobre el enlace covalente y su estructura, encontrando que: un $23 \%$ de los alumnos no considera la influencia de la electronegatividad y la desigual compartición del par de electrones en el enlace polar; un $27 \%$ ve, en la polaridad, un factor que influye en la geometría de las moléculas; un $23 \%$ confunde fuerzas intermoleculares con fuerzas dentro de las moléculas; y un $33 \%$ considera que no existen fuerzas intermoleculares en una red covalente. 
Dumon y Merlin (1988) evalúan conocimientos sobre orbitales moleculares en estudiantes universitarios y encuentran que: $a$ ) la definición de orbital molecular no es bien entendida; $b$ ) el método CLOA parece bien recordado; y $c$ ) las relaciones entre función de onda y función matemática, y de nivel de energía y estado del electrón son bien comprendidas.

Caamaño y Casassas (1987) examinan estudiantes de 16 años y encuentran que: un $50 \%$ no reconocía como elementos sustancias simples formadas por moléculas; un $40 \%$ identificó como moleculares estructuras gigantes; un elevado porcentaje asoció la valencia de un elemento con el subíndice del elemento con que se combina; y la mayoría no sabía calcular el número de enlaces que se rompen y se forman en una reacción química.

De Posada (1999), que revisa las concepciones de alumnos de entre 15 a 18 años encuentra que: los alumnos aceptan la idea que algunas sustancias gaseosas son moleculares; no es bien comprendida la naturaleza de la unión; como así tampoco la naturaleza del enlace covalente; la idea que existen fuerzas intermoleculares ha sido menos interiorizada que el enlace covalente; y la idea de ion no es fácilmente asumida por los alumnos.

Si tenemos en cuenta que la teoría de enlaces y la estructura de las sustancias, junto con aspectos termodinámicos y cinéticos resultan los principios teóricos en los que descansa la química y su enseñanza, que muchos investigadores consideran el concepto de enlace químico como crucial a la hora de desarrollar distintos aspectos de química, física o biología (Benfey, 1965; Allinger et al., 1979; Paoloni, 1979; Solbes y Vilches, 1991) y que tal tema se enfoca a nivel educativo desde la EGB 3 y el ciclo polimodal (alumnos de 12 a 18 años) hasta el nivel universitario, cabe preguntarse entre otras cuestiones: ¿Hay diferencias de concepciones alternativas sobre el tema, entre los alumnos que ingresan a la universidad respecto de aquéllos que ya han recibido instrucción universitaria? ¿Cuáles son esas concepciones? ¿Hay alguna evolución en las mismas? ¿Qué grado de coherencia exhiben los alumnos en sus razonamientos?

Consideramos que el modelo de enlaces químicos pertinente a tal estudio es el que propone, al margen de las fuerzas de atracción entre moléculas, la existencia de tres tipos de enlaces entre los átomos de los elementos: iónico, covalente y metálico, por ser éste, en nuestro país, el único enfocado en el nivel preuniversitario (EGB 3 y ciclo polimodal).

\section{OBJETIVOS}

A los efectos de intentar responder a los interrogantes planteados, juzgamos conveniente desarrollar actividades con los siguientes objetivos:

- Poner de manifiesto las concepciones características de los alumnos respecto a procesos donde se agrupan átomos de un mismo o de distintos elementos.
- Determinar las concepciones referidas a que la posibilidad de una unión química entre átomos sea percibida como un proceso espontáneo.

- Establecer qué concepciones sustentan respecto a la estructura de sustancias unidas por enlace iónico y covalente.

- Investigar las concepciones acerca de la causalidad del enlace iónico.

- Analizar la coherencia, en relación con el modelo teórico aceptado, de las ideas sostenidas por los alumnos.

- Examinar el posible impacto de la enseñanza a nivel universitario sobre las concepciones alternativas de los estudiantes.

\section{METODOLOGÍA}

El estudio se llevó a cabo en forma transversal con dos grupos de alumnos, uno de ingresantes a la universidad y otro de alumnos universitarios. Se utilizó como instrumento de recolección de datos un cuestionario escrito elaborado para la presente investigación, que consta de 15 cuestiones, 13 de opción cerrada y 2 de opción abierta, y que se incluye al final del trabajo como anexo I.

\section{CUESTIONARIO}

Para la elaboración del mismo nos basamos en las transcripciones de una serie de entrevistas semiestructuradas que se mantuvieron, en el marco de la investigación, con alumnos que hacían el Curso de Articulación a Distancia en Química y con quienes cursaban Química General y Química Inorgánica, dentro de la Facultad de Bioquímica y Ciencias Biológicas de la Universidad Nacional del Litoral (UNL). Para tal tarea se tuvo en cuenta las recomendaciones que, sobre la técnica de entrevistas, hacen Osborne y Freyber (1985) y Novak y Gowin (1984).

La lectura de las transcripciones nos permitió elaborar una serie de puntos sobre los que las respuestas de los alumnos parecían diferir respecto de los conceptos sostenidos por expertos en el tema. De acuerdo con dichos puntos se confeccionó una lista de cuestiones que sería interesante enfocar. Éstas se transcriben a continuación:

a) La configuración espacial que adquiere un grupo de átomos unidos por enlaces químicos.

b) La causalidad de un enlace químico.

c) El tipo de unión entre átomos iguales.

d) El tipo de unión entre átomos distintos.

e) La secuencia de fenómenos que ocurren cuando se forma un compuesto iónico a partir de sus elementos. 
f) La atracción mutua entre aniones y cationes.

g) La no-existencia de moléculas en los compuestos iónicos.

h) La posibilidad de formación de moléculas donde se unen átomos iguales.

i) La espontaneidad de los procesos de unión entre átomos iguales.

j) La espontaneidad de los procesos de unión entre átomos distintos.

k) Comparación entre las fuerzas de los enlaces iónicos y covalentes.

l) La no-participación del núcleo atómico en la formación de los enlaces químicos.

Es importante aclarar que los puntos arriba mencionados no agotan las cuestiones que pueden desprenderse del análisis de las transcripciones de las entrevistas; los puntos $e, f, l$, no fueron tratados en esta investigación, ya que no logramos elaborar preguntas lo suficientemente adecuadas.

Una vez evaluadas las ideas a abordar se procedió a la elaboración de las preguntas del cuestionario teniendo en cuenta: los niveles de interpretación y análisis empleados en la ciencia química (macroscópico, microscópico y simbólico) y las características de las concepciones alternativas.

Se intentó que los ítems del cuestionario tuviesen un lenguaje simple, dado que la utilización de términos alejados de la realidad inmediata del alumno conlleva el riesgo de que éste no interprete qué es lo que se le pregunta y, además, porque el uso de un lenguaje demasiado técnico no ayuda a la expresión de las propias ideas de quien responde. Las actividades del cuestionario se elaboraron con enunciados cortos, de modo que su lectura no llevase demasiado tiempo y facilitase que el estudiante retuviera las cuestiones fundamentales de las mismas.

El objetivo de cada pregunta del cuestionario, así como también la clave de respuesta al mismo, se pueden consultar al final del trabajo en el anexo II.

El cuestionario se puso a prueba con dos grupos de estudiantes de la cátedra de Química Inorgánica de la Facultad de Bioquímica y Ciencias Biológicas de la UNL, a los efectos de evaluar si las personas entendían qué cosas se les preguntaban y el tiempo necesario.

\section{MUESTRA}

El instrumento de evaluación se aplicó finalmente a dos grupos de alumnos. El que llamaremos Grupo I estaba integrado por 38 estudiantes que finalizaban el cursado de Química Inorgánica en la Facultad de Bioquímica y
Ciencias Biológicas de la UNL (edades entre 18 y 21 años). El que denominaremos Grupo II estaba compuesto por 46 alumnos que participaban del Curso Común Preparatorio de Ingreso a la UNL (edades entre 17 y 20 años).

\section{TÉCNICAS ESTADÍSTICAS}

El análisis de los resultados se efectuó utilizando el programa SPSS 7.5 para Windows en versión original.

En el estudio de los datos se emplearon tres tipos de prueba:

- Prueba de homogeneidad de proporciones, utilizada para examinar la evolución de las concepciones alternativas.

- Prueba de igualdad para varias proporciones.

- Prueba de independencia, utilizada para examinar la coherencia de las ideas sustentadas por los estudiantes frente a diferentes cuestiones.

Se utilizó un nivel de significancia estadística $\alpha$ de 0,05 en todas las pruebas.

Al procurar determinar la homogeneidad o no de los grupos respecto a sus concepciones alternativas no se consideró, en ningún caso, a aquellos individuos que no respondieron a la pregunta en cuestión.

Sólo se informa del número de individuos que no responden si la cantidad en el grupo es mayor que cinco.

En aquellas preguntas que requieren algún tipo de explicación no se tomará en cuenta a quienes, no eligiendo alternativa alguna en forma explícita, luego la proporcionen.

En la prueba de independencia se adoptó un criterio similar al de las pruebas de homogeneidad en el sentido de no considerar, en el análisis, a aquellos individuos que no respondieron a ambas cuestiones.

\section{RESULTADOS Y COMENTARIOS}

A continuación se detallan, en letra cursiva, las concepciones alternativas detectadas y, posteriormente, los ítems del cuestionario que se utilizaron como reactivos.

- Respecto de procesos donde se agrupan átomos de un mismo o de distintos elementos:

a) El hecho de que dos átomos de un mismo o de distintos elementos se unen formando una molécula es un proceso de cambio de estado de agregación. Análisis en forma conjunta de los pares de preguntas P1-P2 (GI, $13,9 \%$ y GII, $15,2 \%$ ), P4-P5 (GI, $8,6 \%$ y GII, $6,8 \%$ ). 
b) Átomos de un mismo elemento se unen por enlace iónico. Dicha cuestión fue revisada en las preguntas: 3.1 (GI, $16 \%$ y GII, $36,8 \%$ ), 8 -c (GI, $10,5 \%$ y GII, 30,4\%), 9-a (GI, 13,5\% y GII, 30,2\%), 10 (GI, 44,7\% y GII, 45,7\%).

c) Átomos de distintos elementos se unen solamente por enlace iónico. Ítems: 6,1 (GI, 32,1\% y GII, 61,1\%), 9-c (GI, $41,7 \%$ y GII, 51,2\%).

d) La unión de átomos de oxígeno para formar una molécula es un proceso físico. Se evaluó dicha cuestión en las preguntas: 8-a (GI, 5,3\% y GII, 17,4\%), 8-b (GI, $5,3 \%$ y GII, $21,8 \%$ ).

e) El enlace iónico es más fuerte que el enlace covalente. Análisis de las explicaciones de los estudiantes a la pregunta 10 (GI, 5,6\% y GII, 15,4\%). Otra explicación del mismo ítem, en la que especulamos que subyace idéntica concepción, es la de quienes indican que los compuestos con enlace iónico son más duros que aquéllos con enlace covalente (GI, 5,6\%) ignorando la existencia de cristales covalentes. Un conjunto importante de ambos grupos no da explicación alguna (GI, 36,1\% y GII, 41\%).

- Respecto a la posibilidad de que una unión química entre átomos sea percibida como un proceso espontáneo:

Para que átomos de un mismo o diferentes elementos se unan por enlace químico es imprescindible suministrar energía. Tales procesos no se conciben como espontáneos. Los ítems a través de los que se investigó fueron: 3.2 (GI, $52 \%$ y GII, $84,2 \%$ ), 8-d (GI, $21,1 \%$ y GII, 26,1\%), 9-b (GI, 16,2\% y GII, 39\%), 6.2 (GI, 57,6\% y GII, 67,4\%).

- Respecto a la estructura de sustancias unidas por enlace iónico y covalente:

a) Cuando dos átomos del mismo o diferentes elementos se unen por enlace químico, para formar una molécula, permanecen sin modificar en absoluto su estructura interna, como si sólo se hubiesen acercado. Cuestiones: 3.3 (GI, 45,8\% y GII, 58,8\%), 6.3 (GI, 43,3\% y GII, 51,5\%).

b) Al unirse por enlace químico para formar una molécula diatómica, átomos de un mismo o de distintos elementos unen sus núcleos. Ítems: 3.3 (GI, 12,5\% y GII, $17,7 \%), 6.3$ (GI, 26,7\% y GII, 21,2\%).

c) Existen moléculas de sustancias iónicas. Detectada por análisis de las preguntas a los ítems 8-a (GI, 5,3\% y GII, 17,4\%), 9-a (GI, $13,5 \%$ y GII, $30,2 \%$ ) y 11 (GI, $26,3 \%$ y GII, 37,0\%).

- Referido a las causas del enlace iónico:

a) La causa del enlace es la naturaleza misma de los átomos involucrados (metal-no metal), llegando inclusive a adjudicar cierta intencionalidad al comportamiento de uno de los elementos implicados. Pregunta 7 (GI, 38,9\% y GII, 53,7\%).

b) La causa del enlace iónico es que cada uno de los elementos completará su octeto electrónico. Pregunta: 7 (GI, $13,9 \%$ y GII, 14,6\%).
En ambos grupos se investigó la coherencia de las ideas con un test de independencia. Los integrantes de los dos grupos responden en forma poco coherente la mayoría de los catorce pares de cuestiones relacionadas entre sí. Ello pone de manifiesto que, aparte de no existir coherencia en sus interpretaciones, tampoco hay una evolución positiva en tal sentido.

Los pares de cuestiones analizadas y el tema a que se refieren las mismas se detallan a continuación (en negrilla aquéllas en las que, para alguno o ambos grupos, las ideas expuestas ante cuestiones diferentes son similares):

a) Tipo de enlace que existe entre átomos de un mismo elemento: P3.1-P8.c; P3.1-P9.a; P8.c-P9.a (GII); P10P3.1; P10-P8.c; P10-P9.a (GI).

b) Tipo de enlace que existe entre átomos de elementos distintos: P6.1-P9.c.

c) Viabilidad de la unión entre átomos de un mismo elemento como un proceso espontáneo: P3.2-P8.d; P3.2P9.b; P8.d-P9b (GI y GII).

d) Formación de molécula de oxígeno, ¿proceso físico o químico?: P8.a-P8.b.

e) Existencia de moléculas en las sustancias unidas por enlace iónico: P11-P8.c; P11-P9.a; P8.c-P9.a (GII).

El impacto de la enseñanza universitaria sobre las concepciones alternativas de los estudiantes fue examinada a través de un test de homogeneidad. Sólo, en cinco de las veinte cuestiones analizadas, las ideas de los estudiantes universitarios parecen diferir de aquéllas que sustentan los alumnos ingresantes.

Aquellas preguntas en que las muestras resultan no homogéneas y provenientes, por tanto, de poblaciones con ideas diferentes, se detallan a continuación: P1; P2; P8-a; P8-c; P9-b.

\section{CONCLUSIONES}

A continuación se presenta un resumen de las concepciones alternativas detectadas en los grupos de individuos analizados:

1) Respecto a procesos donde se agrupan átomos de un mismo o de distintos elementos:

- El hecho de que dos átomos del mismo o de distintos elementos se unen formando una molécula es un proceso de cambio de estado de agregación.

- Átomos de un mismo elemento se unen por enlace iónico.

- Átomos de distintos elementos se unen solamente por enlace iónico.

- La unión de átomos de oxígeno para formar una molécula es un proceso físico.

- El enlace iónico es más fuerte que el enlace covalente. 
2) Con relación a la posibilidad de que una unión química entre átomos sea percibida como un proceso espontáneo:

- Para que átomos de un mismo o diferentes elementos se unan por enlace químico es imprescindible suministrar energía. Tales procesos no se conciben como espontáneos.

3) En referencia a la estructura de sustancias unidas por enlace iónico y covalente:

- Cuando dos átomos del mismo o de diferentes elementos se unen por enlace químico, para formar una molécula, éstos permanecen sin modificar en absoluto su estructura, como si sólo se hubiesen acercado.

- Al unirse por enlace químico para formar una molécula diatómica, átomos de un mismo o de distintos elementos unen sus núcleos.

- Existen moléculas de sustancias iónicas.

4) En cuanto a las causas de un enlace iónico:

- La causa del enlace es la naturaleza de los átomos involucrados (metal-no metal), llegando inclusive a adjudicar cierta intencionalidad al comportamiento de uno de los elementos implicados.

- La causa del enlace iónico es que cada uno de los elementos completará el octeto electrónico.

Si consideramos la coherencia de las ideas sustentadas por los alumnos frente a distintas preguntas -hecho que fue analizado mediante un test de independencia-, en la mayoría de los casos, no hay concordancia entre lo que contestan los individuos evaluados, aun frente a actividades que hacen referencia a un mismo hecho, dispuestas una a continuación de la otra en el instrumento de evaluación, pero estructuradas de modo diferente. Esto confirmaría la hipótesis de otros investigadores (Driver, 1988) de que las concepciones alternativas se activan o no según el contexto en que el sujeto razone. En un mismo individuo convergen diferentes ideas sobre un mismo tema, las que parecen guardar poca o ninguna relación.

Respecto al impacto de la enseñanza a nivel universitario, de las veinte cuestiones analizadas en sólo cinco de ellas (1/4 del total), las respuestas de los alumnos universitarios fueron significativamente distintas de las de los alumnos ingresantes. En el resto queda convalidada estadísticamente la hipótesis de homogeneidad de las muestras, por lo que la proporción de las distintas ideas presentes en ambos grupos resulta similar. Esto último nos permite concluir que las concepciones alternativas de los individuos no han sido modificadas en grado significativo por la instrucción recibida.

Si bien se cree necesario revisar las prácticas de enseñanza en el ámbito universitario a efectos de considerar si alguna de ellas favorece la presencia de distintas concepciones, tal resultado confirma la persistencia, pese a la instrucción, de las concepciones alternativas.

\section{CONSIDERACIONES FINALES E HIPÓTESIS ABIERTAS}

Las distintas concepciones alternativas encontradas y los análisis estadísticos practicados nos llevan a efectuar las siguientes reflexiones.

En ambos grupos, a nuestro juicio, existe una comprensión deficiente respecto de la estructura que adquieren los átomos al unirse por enlace químico para formar una nueva sustancia. La noción de molécula parece haber sido asimilada por el uno y el otro, aunque a veces la utilicen de modo incorrecto; pero no ocurre lo mismo con la idea de una estructura reticular, tanto para sustancias que se unen por enlace covalente como para aquéllas que lo hacen por enlace iónico. Respecto a estas últimas, si bien frente a algunas actividades los estudiantes universitarios en su mayoría abandonan una imagen molecular de las mismas, las respuestas obtenidas plantean cierta incertidumbre, que la presente investigación no permite dilucidar, sobre si la representación adoptada es compatible o no con el modelo que la ciencia admite.

Coincidimos con De Posada en la poca relación que los estudiantes establecen entre la estructura o representación de una sustancia y su tipo de enlace, pese a que las proporciones que hallamos son diferentes.

Especulamos que ello podría deberse a que, en la enseñanza de la química, cuando se hace referencia a una sustancia y a fin de representarla, se recurre mayoritariamente a su fórmula química y no a la estructura espacial característica de la misma. Si se observa una de tales fórmulas no es difícil imaginar que los estudiantes extrapolen la relación mínima en que deben combinarse los átomos a la disposición real que presentan éstos en la sustancia. Tal razonamiento se vería reforzado porque muchas de ellas se corresponden efectivamente con unidades estructurales de distintos compuestos $\left(\mathrm{H}_{2}, \mathrm{O}_{2}\right.$ $\mathrm{H}_{2} \mathrm{O}$, etc.).

Podría objetarse que generalmente la fórmula va acompañada de una explicación que evita una interpretación errónea, a lo que oponemos la importancia que la percepción tiene en la adquisición de conceptos desde la infancia, sobre todo cuando tales imágenes son reiteradas a lo largo de la enseñanza. No consideramos que tal hecho haya perdido vigencia en el nivel educativo superior; esto queda reflejado al analizar las respuestas de los estudiantes universitarios, en esta investigación, a preguntas sobre sustancias cuya estructura a nivel microscópico guarda algún tipo de similitud.

En cuanto a los distintos tipos de enlace, los alumnos parecen sobrevalorar la importancia del enlace iónico, lo que se evidencia al proponerlo entre átomos que no se ajustan a las características requeridas para que éste sea viable y al considerar que los átomos de distintos elementos se pueden unir exclusivamente por tal tipo de enlace.

Se podría especular que la causa de ello reside en una comprensión deficiente del enlace covalente y de las 
características de las sustancias unidas por este tipo de enlace, razón por la cual no lo utilizan en sus explicaciones.

La falta de entendimiento respecto al mismo podría adjudicarse a la presentación que los libros de texto y los propios docentes hacen del tema de los enlaces químicos. En ella, en general, queda claro que, en un enlace iónico, son las fuerzas electrostáticas las que ocasionan la unión química. En cambio, da la impresión, dado que «los enlaces covalentes se generan por compartición de electrones», que fuerzas extrañas y de carácter desconocido son las que ocasionan el referido tipo de unión. Obviamente tal imprecisión no contribuye a que quien aprende adquiera una noción clara del tema.

Pese a que el enlace iónico parece ser mejor comprendido que el enlace covalente, no hay una interpretación correcta de la estructura de las sustancias iónicas, ni de las razones que conducen a la formación de compuestos estables. Respecto al último punto, en al menos la mitad de ambos grupos, persisten ideas ingenuas o que no explican en realidad las causas de la formación de tales compuestos.

Además, estudiantes del GI y del GII consideran que el enlace iónico es más fuerte que el enlace covalente, confundiendo quizá la dureza de las sustancias iónicas con la fuerza de la unión química. Esta conclusión refuerza la idea expuesta anteriormente sobre la relevancia de lo que el alumno percibe a nivel macroscópico, a través de sus sentidos, respecto de lo que imagina a nivel microscópico.

En cuanto a la formación de sustancias a partir de los elementos que las constituyen, alumnos de ambos grupos creen que tal hecho no es un proceso químico. Si se toma en consideración el contexto en que tales creencias se manifiestan, con sustancias utilizadas en las explicaciones de distintas disciplinas, cabe preguntarse si términos tan fundamentales como proceso físico y proceso químico no son «aprendidos» de modo acrítico por los estudiantes. Los alumnos los utilizan sin comprender el significado que éstos poseen para la comunidad científica, careciendo de noción de los atributos de tales conceptos y, por tanto, cuando deben tomar decisiones basadas en estos últimos aplican criterios que difieren de los científicos. Tal conclusión es aplicable tanto a los conceptos arriba señalados como al de molécula, lo que es utilizado por los alumnos para sustancias con estructura no molecular.

Muchos alumnos parecen no tener en claro el significado de «espontaneidad de una reacción química», lo que, según la segunda ley de la termodinámica, se debe interpretar como la dirección inherente en la que los sistemas fuera del equilibrio se desplazan sin intervención externa. Los estudiantes dejan ver esta dificultad cuando, ante la propuesta explícita de una reacción espontánea, consideran igualmente imprescindible algún tipo de influencia externa.

Por otra parte, la falta de coherencia en las respuestas puede relacionarse con una estructura cognitiva donde los conceptos no están fuertemente conectados entre sí y donde no hay una adecuada generalización de los nuevos conocimientos incorporados por el individuo. Posiblemente también se puede vincular tal hecho con la persistencia, en los alumnos, del aprendizaje memorístico.

Con relación al porcentaje de alumnos que no responden, a las explicaciones solicitadas o alguna pregunta de opción abierta del cuestionario, se podría juzgar a partir de la exigencia de elaborar el propio argumento, pero tal comportamiento se observa también con ítems de opción cerrada, lo que nos induce a desechar tal suposición.

El logro de un cambio o evolución de las concepciones alternativas precedentes no involucra, en nuestra opinión, sólo la sustitución o modificación de ciertos conceptos por otros. La incoherencia en las respuestas de un mismo alumno respecto a distintos sucesos cuyo trasfondo es similar nos inducen a pensar que las concepciones halladas son verdaderas construcciones situacionales, elaboradas a partir de ciertas unidades de información y ciertas estructuras asociativas. Por tanto, su modificación debería ir acompañada de un cambio en los procesos y representaciones mediante los que los alumnos abordan las situaciones a que los enfrentamos.

Finalmente no se puede dejar de mencionar que la transposición didáctica del paradigma del enlace químico, en que se basó este trabajo, está dificultada por el mismo modelo. Es de esperar que en los próximos años los químicos seamos capaces de generar una nueva estructura conceptual que trate de explicar de un modo más simple que la mecánica cuántica es algo tan complejo como las uniones químicas. 


\section{REFERENCIAS BIBLIOGRÁFICAS}

ALLINGUER, N. L. et al. (1979). Química orgánica. Barcelona: Reverté.

AUSUBEL, D. P., NOVAK, J. D. y HANESIAN, H. (1978). Educational Psychology, 2a. ed. Nueva York: Holt, Rinehart y Winston. Trad. de Sandoval, M. (1983). Psicología Educativa. Trillas: México.

CAAMAÑO, A. y CASASSAS, E. (1987). La comprensión de la estructura de la materia y del cambio químico en estudiantes de 15 y 16 años. Enseñanza de las Ciencias, núm. extra, pp. 159-160.

DE POSADA, J. M. (1999). Concepciones de los alumnos sobre el enlace químico antes, durante y después de la enseñanza formal. Problemas de aprendizaje. Enseñanza de las Ciencias, 17 (2), pp. 227-245.

DRIVER, R. (1988). Un enfoque constructivista para el desarrollo del currículo en ciencias. Enseñanza de las Ciencias, 6 (2), pp. 109-120.

DUMON, A. y MERLIN, A. (1988). Dificulties with molecular orbitals. Education in Chemistry, 25 (2), pp. 49-52.
NOVAK, J. y GOWIN, D. B. (1984). Aprendiendo a aprender. Barcelona: Martínez Roca.

OSBORNE, R. y FREYBERG, P. (1985). El aprendizaje de las ciencias. Implicaciones de la ciencia de los alumnos. Madrid: Narcea.

PAOLONI, L. (1979). Toward a culture-based approach to chemical education in secondary schools: The role of chemical formulae in the teaching of chemistry. European Journal of Science Education, 1(4), pp. 365-377.

PETERSON, R.F. y TREAGUST, D.F. (1989). Grade-12 student's misconceptions of covalent bonding and structure. Journal of Chemical Education, 66(6), pp. 459-460.

POZO, J. I. (1987). Aprendizaje de la ciencia y pensamiento causal. Madrid: Visor.

POZO, J. I. (1989). Teorías cognitivas del aprendizaje. Madrid: Morata.

SOLBES, J. y VILCHES, A. (1991). Análisis de la introducción de la teoría de enlaces y bandas. Enseñanza de las Ciencias, 9(1), pp. 53-58. 


\section{ANEXO I}

El cuestionario que te presentamos no es de modo alguno una evaluación para las cátedras de química. Te pedimos que nos ayudes respondiéndolo porque queremos conocer más sobre tus ideas respecto de tal asignatura.

Es por ello que te rogamos que respondas a cada cuestión de modo sincero y reflejando lo mejor que puedas tus ideas.

La encuesta es anónima, pero necesitamos que nos facilites algunos datos a fin de poder trabajar mejor. Por favor, no los omitas.

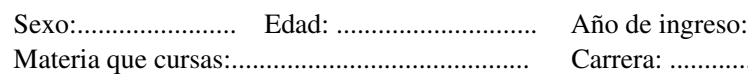

¿En la escuela media diste alguna vez química?:

¿Cuántos años?:

La escuela media de que egresaste era:

Pública Privada

(Tacha lo que no corresponda.)

Terminalidad con la que egresaste de la escuela media (secundario):

Muchas gracias por tu colaboración.

1. La figura 1 es la representación del estado inicial de un sistema, donde $\mathrm{X}$ son átomos de un mismo elemento. Suponiendo que ocurra una transformación espontánea, ¿cuál o cuáles de las siguientes figuras (a o b) crees tú que sería la representación final del mismo?

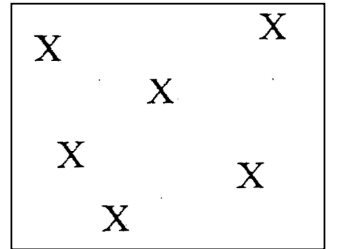

Figura 1 (representación inicial)

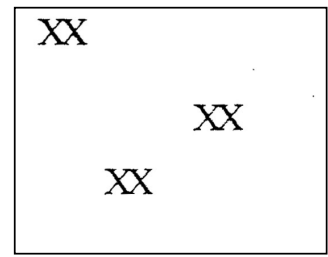

$\boldsymbol{b}$

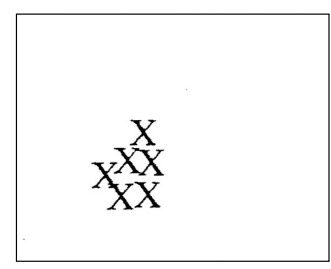

Respuesta:

2. ¿Qué crees que ocurrió, según tu criterio, en el pasaje espontáneo del sistema de la figura 1 del ítem anterior a la representación final (o las representaciones finales) que elegiste? (Marca con una cruz la respuesta correcta.)

a. Cambio de estado de agregación.

a.1. ¿Qué cambio es? :

b. Enlace químico.

c. Otra cosa (Acláranos, por favor, qué.)

Si elegiste el ítem a, pasa a la pregunta 4. Si elegiste el ítem b, pasa a la pregunta 3. Si elegiste el ítem c, pasa a la pregunta 4.

3.1. En el enlace que tú crees que ocurrieron en 2.b: (Marca con una cruz la respuesta correcta.)

a. Los átomos de $\mathrm{X}$ tienen que adquirir cargas de distinto signo para unirse.

$b$. No es necesario que los átomos de $\mathrm{X}$ adquieran cargas de distinto signo para unirse.

c. Depende de cuál sea la identidad de X para determinar si adquieren o no cargas de distinto signo para unirse. 
3.2. ¿Crees que, para que la unión de tales átomos se produzca, es absolutamente necesaria alguna de las siguientes condiciones? De ser así, por favor, indícanos cuál. (Marca con una cruz la respuesta correcta.)

a. Chispa eléctrica

b. Calentar

c. Ionizar

$d$. Dar energía al sistema; si no, los átomos no se unen.

e. Nada.

f. Otros (Acláranos que debería pasar.)

Explícanos, con tus palabras, tu respuesta.

3.3. Si pones en contacto en un recipiente átomos de un mismo elemento (situación representada en el dibujo 1, donde cada esfera de puntos representa a un átomo y el círculo negro central, su núcleo), ¿cuál crees que es la mejor representación de tal sistema luego de una reacción química?

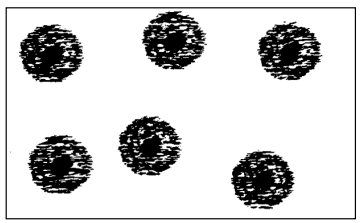

Dibujo 1 (Situación inicial)

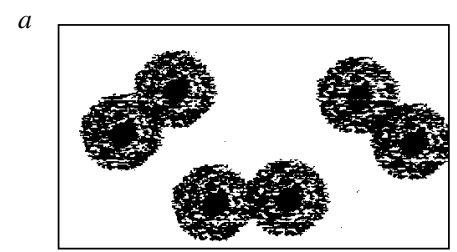

$b$
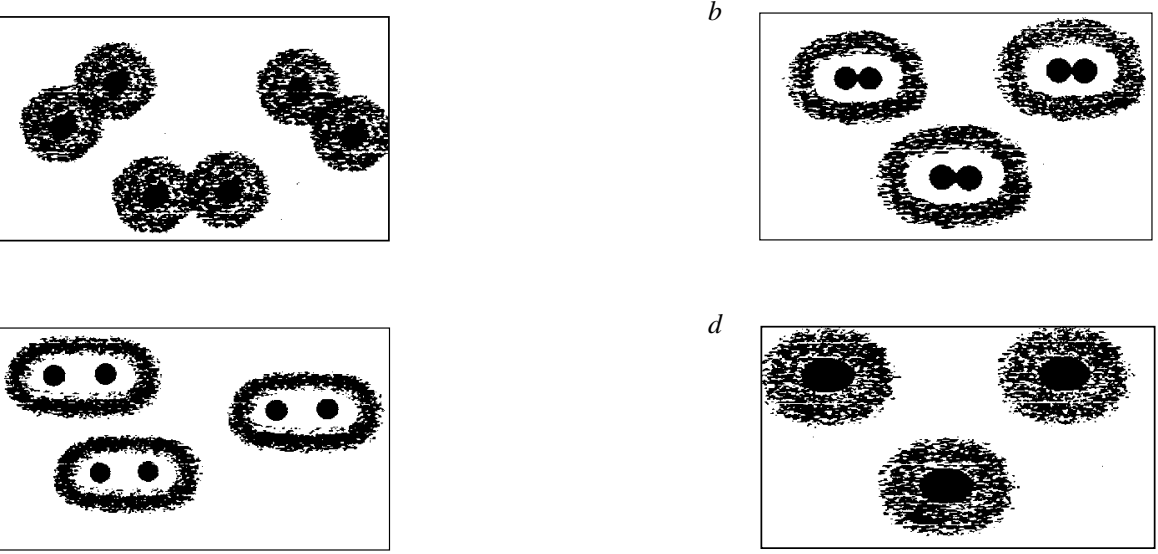

Explícanos, con tus palabras, tu opción.

Sigue ahora con la pregunta 4.

4. Si tienes un sistema como el de la figura 1, donde A representa a átomos de un elemento y B a átomos de otro elemento distinto y tal sistema sufre una transformación espontánea, ¿cuál o cuáles de las siguientes crees tu que sería la representación final del mismo?

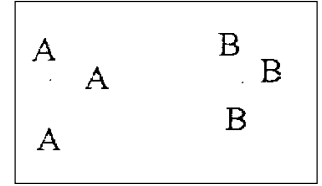

Figura 1

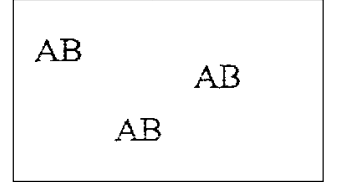

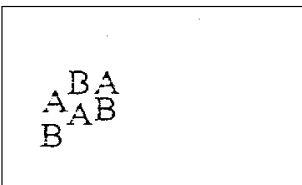

Respuesta: 


\section{INVESTIGACIÓN DIDÁCTICA}

5. ¿Qué crees que ocurrió, a tu criterio, en el pasaje espontáneo del sistema 1 a la representación final (o las representaciones finales) que elegiste? (Marca con una cruz la respuesta correcta.)

a. Cambio de estado de agregación.

¿Cuál cambio?

b. Enlace químico.

c. Otra cosa (Acláranos, por favor, qué.)

Si elegiste el ítem a, pasa a la pregunta 7. Si elegiste el ítem b, pasa a la pregunta 6. Si elegiste el ítem c, pasa a la pregunta 7.

6.1. En el enlace que tú crees que ocurrió en 5.b.: (Marca con una cruz la respuesta correcta.)

a. Los átomos de A y B tienen que adquirir cargas de distinto signo para unirse.

$b$. No es necesario que los átomos de A y B adquieran cargas de distinto signo para unirse.

c. Depende de cuál sea la identidad de los átomos de A y B para determinar si adquieren o no cargas de distinto signo para unirse.

6.2. ¿Crees que, para que el enlace químico de tales átomos se produzca, es absolutamente necesaria alguna de las siguientes condiciones? De ser así, por favor, indícanos cuál. (Marca con una cruz la respuesta correcta.)

a. Chispa eléctrica.

b. Calentar.

c. Ionizar.

d. Dar energía al sistema; si no, los átomos no se unen.

e. Nada.

$f$. Otros (Acláranos qué debería pasar.)..

Explícanos con tus palabras tu respuesta....

6.3. Si pones en un recipiente átomos de dos elementos distintos (situación representada en el dibujo 1, donde cada esfera de puntos con el círculo central negro representa a átomos de un elemento con su núcleo y cada esfera de puntos con el círculo central blanco representa a átomos de otro elemento distinto con su núcleo), ¿cuál crees tú que sería la mejor representación de tal sistema luego de una reacción química?

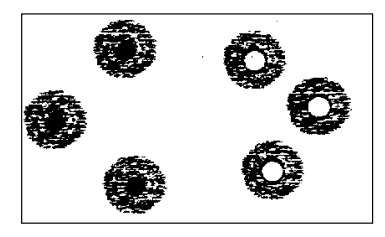

Dibujo 1 (Situación inicial)

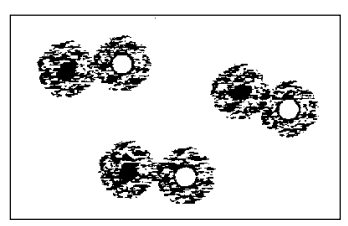

$a$

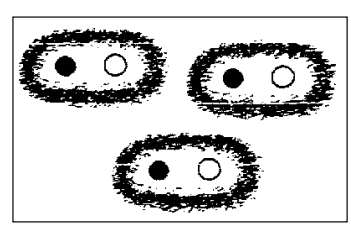

b

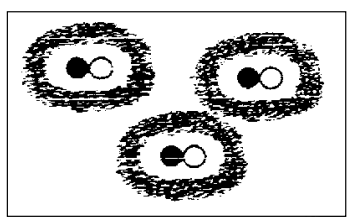

c

Respuesta:

Explícanos tu opción:

Si crees que ninguna de estas opciones corresponde a una respuesta correcta, haz tu propia representación y explícala.

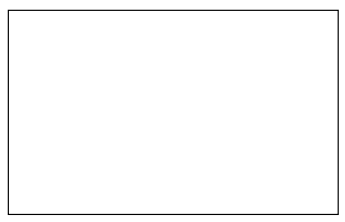

Representación:

Sigue ahora con la pregunta 7. 
7. El yodo y el plomo forman una sal de color amarillo estable a temperatura ambiente: el yoduro de plomo (II). ¿Por qué crees que estos dos elementos se atraen y finalmente se unen formando una sal? (Marca con una cruz la opción correcta.)

a. Porque el yodo es un no-metal y el plomo, un metal, y es natural que se atraigan.

b. Al unirse van a completar cada uno su octeto.

c. Forman una red cristalina iónica.

$d$. Al unirse, el yodo completa su octeto y el plomo cumple su misión cediéndole sus electrones.

8. Tachar lo que no corresponda.

a. Cuando se forma una molécula de oxígeno a partir de dos átomos ocurre un proceso (físico - químico).

b. Para formar dicha molécula, los átomos de oxígeno (se suman - se condensan - se agrupan - se enlazan químicamente).

c. Para formar dicha molécula los átomos (deben - no deben) adquirir cargas de distinto signo.

$d$. Todo lo antes dicho (puede ocurrir - no puede ocurrir) espontáneamente, ya que el sistema ( evoluciona solo - debe recibir energía).

9. Responde con Verdadero o Falso

a. Para que dos átomos de un mismo elemento se unan formando una molécula, deben primero transformarse en iones de distinto signo.

$b$. Para que se unan átomos de un mismo elemento, es necesario siempre proporcionar energía. Tal unión no puede ser, entonces, un proceso espontáneo.

c. Para que dos átomos de elementos diferentes se unan, deben siempre primero transformarse en iones de distinto signo.

10. El diamante (el material más duro que se conoce) y el grafito (mina de los lápices) están formados sólo por átomos de carbono. ¿Crees que las diferencias entre ambos pueden explicarse por qué, en el grafito, los átomos de carbono están unidos por enlace covalente y, en el diamante, los átomos de carbono están unidos por enlace iónico?

$$
\text { Sí No (Tacha lo que no corresponda.) }
$$

Explícanos con tus propias palabras tu respuesta.

11. La notación $\mathrm{H}_{2} \mathrm{O}$ se lee «una molécula de agua». ¿Cómo se lee la notación $\mathrm{NaCl}$ ? 


\section{ANEXO II}

Análisis de las preguntas del cuestionario ANEXO I

\section{Pregunta 1}

Objetivos: a) Determinar las ideas de los alumnos sobre el estado final que alcanza espontáneamente un sistema formado inicialmente por átomos aislados de un mismo elemento. $b$ ) Analizar si conciben todas las posibilidades frente a la situación planteada, como lo haría un experto, o sólo alguna de ellas.

La cuestión se presenta en un plano abstracto al no indicarse la identidad de los átomos. Su elección responde a que, en química, se pretende que el alumno acceda a razonar en un plano ideal sobre construcciones mentales que interpretan la realidad; y es en tal nivel donde parece pertinente averiguar qué concepciones tiene.

Por transformación espontánea se interpreta el cambio que tiene tendencia natural a producirse sin ser impulsado por una influencia externa en un sistema que no está en equilibrio. En este ítem, la transformación puede ser física o química y en el cuestionario no se da indicación para desechar uno u otro.

La pregunta se aparta de aquellas situaciones que se proponen de modo usual en clase para eludir una respuesta «de memoria» $\mathrm{o}$ «aprendida» $\mathrm{y}$ provocar una réplica personal. Por ello se parte de una situación poco común y, por tanto, altamente desestructurante, como la de presentar como estado inicial un sistema formado por átomos aislados.

\section{Análisis de las respuestas posibles}

La respuesta correcta es la opción de ambos estados finales, $\boldsymbol{a}$ y $\boldsymbol{b}$. Una opción por alguno en forma individual sería parcialmente correcta.

El razonamiento que suponemos que realiza quien elige la opción a es pensar en la representación de una sustancia simple que forma moléculas biatómicas (como el $\mathrm{N}_{2}, \mathrm{O}_{2}$, etc.).

Si elige la opción b, podría pensar en la representación de un cristal covalente (como el diamante o el grafito) o podría considerar que se trata de moléculas poliatómicas de sustancias simples (como el $\mathrm{S}_{8}$ o el $\mathrm{P}_{4}$ ), aunque la representación no coincide con ellas. También podría suponer que ocurrió un cambio de estado de agregación.

La pregunta 1 da una idea sobre si el alumno concibe todas las posibilidades frente a la situación planteada o mantiene una visión parcial; pero su valoración completa requiere considerarla en conjunto con la pregunta 2, donde se pide aclaración sobre si el tipo de cambio ocurrido es físico o químico.

No todos los pares de opciones (pregunta 1 - pregunta 2) resultan correctas; ciertas duplas, que en forma separada no indican nada en particular, podrían corresponder a concepciones alternativas, analizadas en forma conjunta.

\section{Pregunta 2}

Objetivo: que el alumno aclare si considera que la transformación planteada en la pregunta 1 se debe a un proceso químico, físico o ambos

\section{Análisis de las respuestas posibles}

La respuesta correcta es la elección conjunta de las alternativas a y b, acompañando a la primera una explicación que indique el pasaje de un estado de agregación menos condensado a uno más condensado. La elección de una sola alternativa es parcialmente correcta.

Si elige sólo la alternativa b, no ha imaginado la posibilidad de un cambio de estado de agregación. Si elige sólo la alternativa a, no concibe una reacción química entre átomos de un mismo elemento.

El análisis conjunto de las preguntas 1 y 2 permite concebir las siguientes categorías:

- Categoría 1 (P1 a y b; P2 a y b): concibe toda la gama de alternativas frente al hecho propuesto; no puede asegurarse si el alumno piensa que sólo frente a la alternativa 1-b es correcto suponer que la evolución del sistema se debe a un cambio de estado de agregación.

- Categoría 2 (P1 a y b; P2 b): no ha considerado la posibilidad de un cambio de estado de agregación pero correctamente ha tenido en cuenta que a los estados finales a y b de la pregunta 1 se puede arribar por el enlace químico entre los átomos. Probablemente conciba la formación de moléculas biatómicas y cristales covalentes entre átomos de un mismo elemento.

- Categoría 3 (P1 a; P2 b): no ha analizado la posibilidad de un cambio de estado de agregación. Tampoco tiene en cuenta que un enlace químico puede llevar al estado final 1-b. Posiblemente conciba la formación de moléculas biatómicas entre átomos de un mismo elemento; pero en el mismo contexto no imagina la formación de cristales covalentes. 
- Categoría 4 (P1 b; P2 b): no ha analizado la posibilidad de un cambio de estado de agregación. Tampoco tiene en cuenta que un enlace químico puede llevar al estado final 1-a. Posiblemente conciba la formación de un cristal covalente; pero en el mismo contexto no imagina la formación de moléculas biatómicas entre átomos de un mismo elemento.

- Categoría 5 (P1 b; P2 a y b): ha analizado que tanto la posibilidad de un cambio de estado de agregación como la de un enlace químico puede llevar al estado final $1 \mathrm{~b}$, pero no considera que un enlace químico puede dar lugar al estado final (se cambió la palabra desembocar). Posiblemente concibe la formación de cristales covalentes pero no la formación de moléculas biatómicas en el mismo contexto.

- Categoría 6 (P1 a; P2 a y b): ha analizado tanto la posibilidad que ocurra un enlace químico entre los átomos como que se verifique un cambio de estado de agregación, pero incorrectamente relaciona este último con el estado final 1-a. Probablemente conciba la formación de moléculas diatómicas por enlace químico entre los átomos, pero en apariencia cree que también un cambio de estado de agregación puede conducir a las mismas.

- Categoría 7 (P1 b; P2 a): no contempla la posibilidad que ocurra un enlace químico entre átomos de un mismo elemento. La única posibilidad evidente para quien responde es un cambio de estado de agregación, el que correctamente relaciona con la configuración 1-b.

- Categoría 8 (P1 a y b; P2 a): no contempla que ocurra un enlace químico entre átomos de un mismo elemento. La única posibilidad evidente para quien responde es un cambio de estado de agregación. La respuesta, aparentemente correcta, se invalida, pues cree que tal transformación puede llevar a los átomos a unirse por pares.

- Categoría 9 (P1 a; P2 a): no contempla la posibilidad de que ocurra un enlace químico entre átomos de un mismo elemento. El cambio de estado que predice es inconsistente.

\section{Pregunta 3}

Esta serie de preguntas se relaciona con las preguntas 1 y 2, pues se solicita que el alumno aclare distintos puntos si consideró que la evolución del sistema se debía a un enlace químico.

\section{Pregunta 3.1}

Objetivo: determinar qué idea tienen los estudiantes sobre el tipo de enlace, iónico o covalente, que ocurre entre átomos iguales de identidad desconocida.

La pregunta se plantea en un plano abstracto.

\section{Análisis de las respuestas posibles}

La opción correcta es la b. La misma permitiría especular que se piensa en un enlace covalente entre átomos de un mismo elemento, ya sea para formar una molécula diatómica (opción a de la pregunta 1), o una molécula poliatómica, o un cristal covalente (opción b de la pregunta 1).

Las opciones a y c son incorrectas.

La primera porque no se concibe que dos o más átomos de un mismo elemento se transformen en iones de distinto signo al unirse por enlace químico y tal opción permite especular que el alumno lo considera posible.

La segunda porque no es en absoluto necesario conocer la identidad de átomos para saber que no se transforman en iones de signo contrario al unirse por enlace químico.

Tampoco hay formación de iones de diferente signo en el enlace metálico.

\section{Pregunta 3.2}

Objetivos: a) Averiguar si los alumnos comparten con los expertos en química la idea sobre qué representa un proceso espontáneo. $b$ ) De no poseer una concepción acorde con las ideas de la comunidad científica, investigar qué condiciones creen necesarias para que se produzca el enlace químico entre átomos de un mismo elemento.

Esta pregunta se ha planteado en un plano abstracto.

Por proceso natural o espontáneo se entiende todo aquel cambio que sucede en un sistema que se abandona a su suerte y llega finalmente a algún estado de equilibrio. Por estado de equilibrio se entiende aquél que, en un sistema cerrado, se caracteriza porque sus variables intensivas (presión, temperatura, composición) tienen un valor que no se altera en el tiempo. 
INVESTIGACIÓN DIDÁCTICA

\section{Análisis de las respuestas posibles}

Tomando en consideración la relación existente entre esta cuestión y la pregunta 1, donde se aclara específicamente que el proceso sucede en forma espontánea, la respuesta correcta es la e.

Cualquiera de las otras opciones indicaría que el alumno no ha tomado en cuenta la relación entre al pregunta 3-2 y la pregunta 1 o que no hay una correlación entre sus ideas y las de los científicos respeto a qué es un proceso que ocurre espontáneamente.

Idéntica cuestión se aborda en las preguntas 8-d y 9-b. Un análisis conjunto de las mismas pretende demostrar si el alumno mantiene sus ideas (sean éstas coherentes con las científicas o no) o bien activa distintas concepciones según el contexto de aplicación. Esto último prestaría apoyo al enfoque que considera que las concepciones alternativas se ajustan más a una categoría natural o concepto probabilístico que a una categoría lógica y bien definida como son los conceptos científicos.

\section{Pregunta 3.3}

Objetivo: determinar qué concepción tienen los alumnos de la estructura de una molécula diatómica de una sustancia simple (nivel microscópico).

La pregunta se plantea en un plano abstracto.

Se consideró que la representación correcta debía reflejar un cambio en la estructura de la nube electrónica y no un simple acercamiento de dos átomos que mantenían su nube electrónica inalterada. Los núcleos deberían hallarse a menor distancia de la que tendrían si los átomos simplemente se hubiesen acercado hasta tocarse.

Tales premisas se tomaron en cuenta para dibujar la representación c.

Puede que la referida alegoría sea discutible desde diferentes aspectos como todo aquello que trata de mostrar algo que nadie ha percibido directamente en forma visual. Pero creemos que cumple la función de resaltar como la más aproximada a las ideas de los científicos. Las otras representaciones resumen la interpretación que se hizo de las ideas expuestas en las entrevistas previas con los alumnos.

\section{Análisis de las respuestas posibles}

La opción correcta es la c.

La opción a sustenta la idea de que los átomos, al unirse por enlace químico, se acercan hasta estar muy juntos; pero nada en ellos sufre una reestructuración. Probablemente estos alumnos no desconozcan la existencia de partículas cargadas en el átomo y los tipos de interacción entre las mismas pero, aparentemente, cada uno de esos conocimientos parece formar parte de estructuras o redes conceptuales diferentes con poca o ninguna relación entre sí.

La opción b sustenta una reestructuración profunda de los átomos unidos por enlace químico y considera que se modifica tanto la nube electrónica como la distancia entre los núcleos. Pero hay divergencia entre sus concepciones y las de los científicos, ya que éstos no consideran que luego de una reacción química los núcleos se unan.

La opción d sugiere que, en la reacción química, los dos núcleos se unen para formar uno sólo, a la manera de las reacciones de fusión nuclear, con una única nube electrónica en torno a él.

La extrapolación a nivel microscópico de que, cuando dos átomos se unen para formar una sustancia, ésta tiene propiedades diferentes de las de los átomos por separado, podría hacer inducir al alumno a considerar que nada preexiste de la anterior configuración.

\section{Pregunta 4}

Objetivo: determinar qué ideas tienen los estudiantes sobre el estado final que alcanza un sistema de átomos de elementos distintos luego de una transformación espontánea.

El propósito de la pregunta y la justificación de las características del planteo son idénticas a las expuestas al analizar la pregunta 1.

Tanto la pregunta 4 como las preguntas 5, 6.1, 6.2 y 6.3 son duplicado de las propuestas 1, 2, 3.1, 3.2 y 3.3 pero frente a un sistema formado por átomos de dos elementos diferentes, identificados para el caso como A y B. Ello se consideró necesario, pues, si las concepciones de los alumnos son en parte función del contexto en que razona, era útil investigar si había o no diferencias entre lo que consideraban los estudiantes frente al primer grupo de cuestiones respecto de sus opiniones frente al segundo grupo. 


\section{Análisis de las respuestas posibles}

La respuesta correcta es la elección de ambos estados finales, a y b. La opción por alguno de los dos en forma individual es parcialmente correcta.

La opción a sustenta la representación de una sustancia compuesta con moléculas diatómicas (ejemplos: CO, NO, etc.). También podría considerar que se trata de moléculas poliatómicas de sustancias compuestas (como las de $\mathrm{SO}_{2}, \mathrm{H}_{2} \mathrm{O}$, etc.), pese a que el número de átomos representados no coincide con las mismas.

La opción b soporta la representación de un cristal covalente (ejemplos: el cuarzo o el nitruro de boro) aunque la estructura dibujada no coincida con la representación de dichos compuestos. Otra suposición correcta sería que ocurrió un proceso físico de cambio de estado de agregación de dos sustancias simples monoatómicas.

Esta pregunta da una idea sobre si el alumno concibe todas las posibilidades frente a la situación planteada o mantiene una visión parcial; su valoración completa requiere considerarla en conjunto con la pregunta 5, donde se pide aclaración sobre si el tipo de cambio ocurrido es físico o químico.

No todos los pares de opciones (pregunta 4 - pregunta 5) resultan correctas; ciertas duplas, que en forma separada no indican nada en particular, podrían corresponder a concepciones alternativas analizadas.

\section{Pregunta 5}

Objetivo: conocer si el alumno considera que la transformación espontánea planteada en la pregunta 4 se debe a un proceso químico, físico o ambos.

En cuanto al análisis de la cuestión y la justificación de su estructura, resultan en todo similares a lo expuesto respecto de la pregunta 2.

\section{Análisis de las respuestas posibles}

La respuesta correcta es la elección conjunta de las alternativas a y b, acompañando a la primera una explicación que indique que el cambio corresponde al pasaje de un estado menos condensado a uno más condensado. La elección de una sola alternativa es una respuesta parcialmente correcta.

Si elige sólo la alternativa b, no ha imaginado la posibilidad de un cambio de estado de agregación. Si elige sólo la alternativa a, no concibe una reacción química entre átomos de elementos distintos.

En cuanto al análisis conjunto de las preguntas 4 y 5 , los pares de respuestas posibles y las explicaciones de su significado coinciden con los expuestos en el análisis conjunto de las preguntas 1 y 2 , salvo por el hecho que se deben relacionar con átomos de dos elementos distintos.

\section{Pregunta 6}

Esta serie de cuestiones se relaciona con las preguntas 4 y 5, pues se solicita que el alumno aclare distintos puntos si consideró que la evolución del sistema se debía a un enlace químico. El objetivo es similar entre las preguntas: 3.1-6.1; 3.2-6.2 y 3.3-6.3. El análisis de las respuestas es similar entre las preguntas: 3.2-6.2 y 3.3-6.3. Sólo se incluye el análisis de las respuestas a la pregunta 6.1 por presentar alguna diferencia con el de la pregunta 3.1.

\section{Análisis de las respuestas posibles a la pregunta 6.1}

La opción correcta es la b, donde los átomos enlazados no se han transformado en iones.

Tal opción permitiría especular que el alumno piensa en un enlace de tipo covalente donde dos átomos de distintos elementos se unen para formar: una molécula diatómica (opción a de la pregunta 4), o un cristal covalente (opción b de la pregunta 4).

Las opciones a y c son incorrectas.

La primera porque, si bien se puede considerar que al unirse por enlace químico dos átomos de elementos distintos se transformen en iones de diferente signo, al ser la única posibilidad admitida, no se dejaría lugar a la existencia de moléculas de sustancias compuestas.

La segunda porque, teniendo en cuenta las representaciones de la pregunta 4, no es en absoluto necesario conocer la identidad de los átomos para saber que no necesariamente se transforman en iones de signo contrario al unirse por enlace químico. 
INVESTIGACIÓN DIDÁCTICA

\section{Pregunta 7}

Objetivo: determinar qué idea tienen los alumnos sobre las causas de la formación de un enlace iónico.

\section{Análisis de las respuestas posibles}

La opción correcta es la c. El alumno al elegirla no sólo estaría considerando el tipo de enlace que une a los átomos, sino también que un compuesto iónico en estado sólido no se encuentra como una molécula aislada. La formación de la red cristalina es la causa de la estabilidad de la sustancia, lo que determina que la energía del producto sea menor que la de los reactivos que le dieron origen.

Las demás opciones de respuesta son incorrectas.

La opción a, si bien utiliza el concepto de metal y no-metal, lo hace en referencia a una propiedad que determina invariablemente la unión de dichas sustancias, sin alusión alguna a la estructura de las mismas. Es una explicación casi mágica y poco adecuada al nivel educativo que transitan los encuestados de nivel universitario.

La opción b hace uso de la regla del octeto; subyace un carácter finalista en la idea de que los átomos van a completar cada uno su octeto, como si se unieran químicamente con este objetivo.

La opción d no sólo utiliza la regla del octeto, sino que también parece dotar a los átomos de un carácter animista al adjudicar al metal una misión a cumplir.

\section{Pregunta 8}

Esta serie de ítems investiga las ideas de los estudiantes sobre moléculas diatómicas de sustancias simples. Se plantea en un plano concreto, ya que se utiliza una sustancia de identidad conocida y lo más cercana posible a la vida cotidiana de los alumnos.

\section{Pregunta 8-a}

Indaga si los alumnos consideran la formación de una molécula biatómica de una sustancia simple como un proceso físico o químico.

\section{Análisis de las respuestas posibles}

La respuesta correcta es «proceso químico», dado que se modifica la identidad de las sustancias que participan en la transformación, y las unidades microscópicas que intervienen cambian su estructura.

\section{Pregunta 8-b}

Indaga si los estudiantes consideran la formación de una molécula biatómica de una sustancia simple como un proceso físico o químico. Tal cuestión permite analizar la coherencia o no de las ideas de quienes respondan también a la pregunta 8-a.

\section{Análisis de las respuestas posibles}

La opción correcta es «se enlazan químicamente». Todas las demás son incompatibles con el proceso que ocurre al formarse una molécula de oxígeno.

\section{Pregunta 8-c}

Objetivo: analizar si los alumnos consideran que dos átomos de un mismo elemento se unen por enlace iónico.

Se relaciona con las preguntas 3.1 y 9 -a, donde se enfoca el mismo tema en un contexto abstracto.

\section{Análisis de las respuestas posibles}

La opción correcta es tachar la palabra deben, sugiriendo la posibilidad de un enlace covalente. Quienes tachan no deben piensan que dos átomos de oxígeno se han transformado en iones de distinto signo y, probablemente, consideran que tal molécula se ha unido por enlace iónico.

\section{Pregunta 8-d}

Objetivo: determinar si consideran la formación de una molécula entre átomos de un mismo elemento como un proceso espontáneo.

La cuestión se relaciona con las preguntas 3.2 y 9-b, donde se enfoca el mismo tema en un contexto abstracto. 


\section{Análisis de las respuestas posibles}

La respuesta correcta es seleccionar las opciones «puede ocurrir» y «evoluciona solo», ya que se estaría considerando la formación de una molécula de oxígeno como un proceso espontáneo.

Si seleccionan las opciones «no puede ocurrir» y «debe recibir energía», el alumno considera la reacción de formación como un proceso no espontáneo.

Esto no es correcto, ya que el oxígeno existe como molécula en condiciones ambiente, lo que implica una variación de energía interna del proceso negativa y una transformación espontánea. Sin embargo, las ideas sostenidas, aunque incorrectas, son coherentes.

El seleccionar otra combinación cualquiera de las alternativas de respuesta implica que, o bien no ha leído con atención la afirmación que escribe, o sustenta ideas incoherentes entre sí.

\section{Pregunta 9}

Cada ítem persigue una finalidad distinta y el único hilo común es el procedimiento de respuesta que se solicita.

\section{Pregunta 9-a}

Objetivo: determinar si los alumnos consideran que, en la molécula de una sustancia simple, los átomos se unen por enlace iónico. La cuestión se plantea en un plano abstracto.

\section{Análisis de las respuestas posibles}

La respuesta correcta es considerar falsa la proposición, ya que dos átomos que forman una molécula no pueden unirse por enlace iónico. Tanto la unidad estructural propuesta, una molécula, como el tipo de elementos que participan de la unión deberían conducir a rechazar el enunciado.

\section{Pregunta 9-b}

Objetivo: determinar si los alumnos conciben o no la unión entre átomos de un mismo elemento como un proceso espontáneo. El planteo se hace en forma abstracta.

\section{Análisis de las respuestas posibles}

La afirmación que se hace es falsa, ya que excluye en forma expresa como proceso espontáneo a cualquiera que implique la unión de átomos de un mismo elemento.

\section{Pregunta 9-c}

Objetivo: investigar qué tipo de enlace consideran que liga a átomos de elementos distintos. La cuestión se plantea en un plano abstracto y se relaciona con la pregunta 6.1 .

\section{Análisis de las respuestas}

La respuesta correcta es que la afirmación es falsa, pues la misma descarta la existencia de un enlace de tipo covalente entre átomos de elementos distintos. Sin embargo, la pregunta no permite dilucidar si quien responde correctamente considera el enlace covalente la única alternativa posible, lo cual también sería erróneo.

Si responde que la afirmación es verdadera, evidentemente sólo considera posible un enlace donde intervengan iones, lo cual es inconsistente con la existencia de moléculas de sustancias compuestas.

\section{Pregunta 10}

Evalúa en forma directa si consideran consecuencia natural del tipo de enlace que liga a los átomos de una sustancia simple (iónico o covalente) el hecho de que ésta sea más o menos dura.

Las sustancias que se mencionan en el enunciado están formadas únicamente por átomos de carbono. Una respuesta afirmativa indicaría dos concepciones alternativas: por un lado, que átomos de un mismo elemento se unen por enlace iónico y, por otro, que las sustancias que presentan tal tipo de enlace son más duras que aquéllas unidas por enlace covalente. 


\section{INVESTIGACIÓN DIDÁCTICA}

Una idea previa como la expresada en segundo término podría tener como base el siguiente esquema alternativo: el enlace iónico es más fuerte que el enlace covalente. Y es la presencia de tal esquema la que se pretende validar en forma indirecta, mediante las explicaciones a la presente cuestión.

La pregunta se plantea en un plano concreto.

Análisis de las respuestas posibles

La opción correcta es responder «no» a la cuestión, dado que átomos de un mismo elemento no se unen por enlace iónico. De las explicaciones de quienes respondan en forma incorrecta se podrá analizar la existencia o no del esquema alternativo propuesto.

\section{Pregunta 12}

Objetivo: investigar si los alumnos consideran que existen moléculas de sustancias iónicas.

\section{Análisis de las respuestas posibles}

El mismo se debe hacer valorando la explicación que cada alumno brinde a la pregunta. 